\title{
Analysis of a quantum well structure optical integrated device
}

\author{
Sh.M. Eladl and M.H. Saad \\ Radiation Eng. Dept., 3 Ahmed Elzomor St., NCRRT, \\ P.O. Box 29, Nasr City, Atomic Energy Authority, \\ Cairo, Egypt
}

\begin{abstract}
This paper demonstrates theoretical modeling of a quantum well structure optical integrated device. The constituent devices of the developed structure are a Quantum Well Infrared Photodetector (QWIP) to detect the optical infrared signal, a Heterojunction Phototransistor (HPT) to amplify the signal, and a Light Emitting Diode (LED) to emit this signal in a visible form. The model is based on the transient behavior of the constituent parts of the structure. The dominant pole approximation scheme is used to reduce its transfer function. The convolution theorem is used to get the overall transient response of the device under consideration. All interesting parameters concerning the transient response, rise time, output derivatives are theoretically investigated. The results show that the overall transient behavior, output derivative, and rise time of the considered structure are approximately the same as the constituent device possessing the lowest cutoff frequency. This type of model can be applied with high sensitivity in the up conversion of infrared or far infrared range for image signal processing.
\end{abstract}

Keywords: quantum well infrared photodetector, heterojunction phototransistor, light emitting diode, quantum well structure, optical integrated device.

Manuscript received 19.11.16; revised version received 05.04.17; accepted for publication 14.06.17; published online 18.07.17.

\section{Introduction}

For optical image processing applications, it is necessary to focus on devices and components that can detect, process, and transmit information with great adaptability and better efficiency [1]. An effective scheme is developed by integrating of Quantum Well Infrared Photodetector (QWIP) and a Light Emitting Diode (LED) to be used as a pixel detector to far or middle infrared radiation with near infrared or visible output, the structure with this scheme is called as up converter [2].

The QWIP is applied for under IR light illumination where its resistance decreases, which yields the voltage drop across the LED to increase and, therefore, an increase in the output intensity. This device is thus an IR converter [3, 4]. When a forward bias voltage is applied, recombination between the photocurrent electrons from the detector side and the injected holes take place giving rise to an intensified light in the LED side.

To improve the quantum efficiency of the up converter, a bottom mirror for the infrared detector side and a resonant cavity for the emitting side are introduced. In this way, the quantum efficiency could be 5-6-fold increased, and the contrast transfer function is even better in comparison with that of the conventional structure [5]. Other theoretical and experimental investigations for conversion from mid infrared to visible LED (MIR type-I LEDs) were developed. Simulation results show that a good control of the optical wavelength and efficient confinement for carrier in the active region is obtained if a MQW active region in the structure design is included [6]

The improvement of selectivity inherent to these devices can be made by using quantum well infrared photodetectors having a resonator (R-QWIP). The 
benefit of resonances is to increase the quantum conversion efficiency [7-9]. These detectors will convert the far infrared light signal to be amplified by HBT which derive a visible Light Emitting Diode (LED).

The input infrared light signal is applied to the developed quantum well structure as it is converted to photo-excited carriers by the Quantum Well Infrared Photodetector (QWIP); the HBT is used to amplify the QWIP output electric signal; and the LED is driven by this output amplified signal transferred from the HBT and radiates an intensified light of near infrared or visible range. The dynamic response of this structure was analyzed being based on the frequency response of its constituent devices [9].

This paper focuses on examining the closest constituent device of the developed structure to its exact dynamic behavior. The pole approximation scheme and convolution theorem are two useful methods to complete this issue, this analysis is important for designing and deeper understanding this type of structure.

The paper is organized as follows: formulation of the specified parameters that describes the transient response, derivative, and rise time is presented in Section 2. The generated curves as results are outlined and discussed in Section 3. Finally, conclusion of this work and an important note to continue the research of this subject have been discussed in Section 4.

\section{Theoretical analysis}

The schematic layer structure of the device under study is shown in Fig. 1. To understand the transient response of the device version under study, it is important to investigate the transient response of each element that constitutes it. The recognition of the characteristic equation describing the overall transient response becomes available when the transient response equation of each element is known.

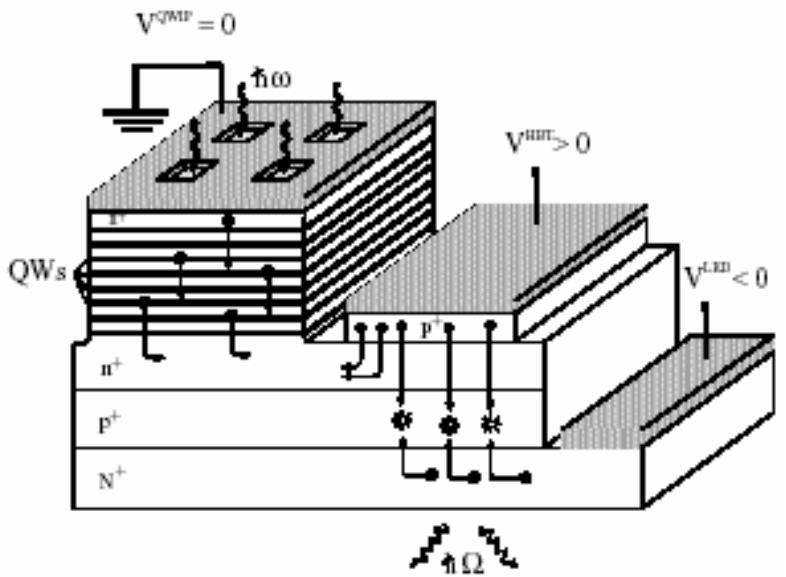

Fig. 1. Schematic structure of an integrated QWIP-HBT-LED pixel.
The relative overall frequency response can be obtained from [10] as follows:

$$
\begin{aligned}
& R(\omega)=\frac{S(\omega)}{\eta_{\mathrm{QWIP}} \eta_{\mathrm{HBT}} \eta_{\mathrm{LED}}}= \\
& =\frac{1}{\left(1+\frac{j \omega}{\omega_{\mathrm{QWIP}}}\right)\left(1+\frac{j \omega}{\omega_{\mathrm{HBT}}}\right)\left(1+\frac{j \omega}{\omega_{\mathrm{LED}}}\right)} .
\end{aligned}
$$

The transfer function of the QWIP-HBT-LED structure has three distinct real roots, it is possible to use useful approximations to reduce the model to the first order system. The most common way to do that is to use the dominant pole approximation scheme. This method is applied to the transfer function in the form of frequency, time or Laplace domain. The dominant pole of the structure means the one that is closer to the origin on Pole-Zero Map which means the slowest pole. This method is restricted to the examination of the transient response and can't be applied to calculate the bandwidth of the structure. The dominant pole approximation scheme can be applied as

$$
R(\omega)=\left\{\begin{array}{l}
R_{\mathrm{QWIP}}(\omega)=\frac{1}{\left(1+\frac{j \omega}{\omega_{\mathrm{QWIP}}}\right)} \text { for } \omega_{\mathrm{QWIP}}<\omega_{\mathrm{HBT}}<\omega_{\mathrm{LED}} \\
R_{\mathrm{HBT}}(\omega)=\frac{1}{\left(1+\frac{j \omega}{\omega_{\mathrm{HBT}}}\right)} \text { for } \omega_{\mathrm{HBT}}<\omega_{\mathrm{LED}}<\omega_{\mathrm{QWIP}} \\
R_{\mathrm{LED}}(\omega)=\frac{1}{\left(1+\frac{j \omega}{\omega_{\mathrm{LED}}}\right)} \text { for } \omega_{\mathrm{LED}}<\omega_{\mathrm{HBT}}<\omega_{\mathrm{QWIP}}
\end{array}\right.
$$

where $\eta_{\mathrm{QWIP}}, \eta_{\mathrm{HBT}}$, and $\eta_{\mathrm{LED}}$ are the conversion efficiency of QWIP, HBT, and LED, respectively. Also, $\omega_{\mathrm{QWIP}}, \omega_{\mathrm{HBT}}$, and $\omega_{\mathrm{LED}}$ are the cutoff frequency of QWIP, HBT, and LED, respectively.

Let $R_{1}(t), R_{2}(t)$, and $R_{3}(t)$ be the relative transient responses at the output side of QWIP, HBT, and LED, respectively. The convolution among them is also a function of time and is denoted by [11].

If the input light is assumed as a unit step time signal, the convolution theorem is applied to the constituent devices as

$$
R_{0}^{\mathrm{QWIP}}(t)=R_{1}(t)=\int_{0}^{t} U(t-\lambda) R_{\mathrm{QWIP}}(\lambda) d \lambda=\left(1-e^{-\omega_{\mathrm{QWIP}} t}\right)
$$

where $U(t-\lambda)$ is a unit step input signal delayed by time $\lambda$ and $R_{\mathrm{QWIP}}(\lambda)$ is $R_{\mathrm{QWIP}}(t)$ at time $\lambda$. 
If the unit input light is inputted to HBT, the individual relative time response for HBT can be get as

$$
R_{0}^{\mathrm{HBT}}(t)=\int_{0}^{t} U(t-\lambda) R_{\mathrm{HBT}}(\lambda) d \lambda=\left(1-e^{-\omega_{\mathrm{HBT}} t}\right),
$$

where $R_{\mathrm{HBT}}(\lambda)$ is $R_{\mathrm{HBT}}(t)$ at time $\lambda$.

At the output side of HBT, one can get

$$
R_{2}(t)=R_{1}(t) \cdot R_{\mathrm{HBT}}(t)=\int_{0}^{t} R_{1}(t-\lambda) R_{\mathrm{HBT}}(\lambda) d \lambda .
$$

The above equation is calculated as

$$
\begin{aligned}
& R_{2}(t)=\frac{1}{\omega_{\mathrm{QWIP}}-\omega_{\mathrm{HBT}}} \times \\
& \times\left\{\omega_{\mathrm{HBT}} e^{-\omega_{\mathrm{QWIP}}}+\omega_{\mathrm{QWIP}}-\omega_{\mathrm{HBT}}-\omega_{\mathrm{QWIP}} e^{-\omega_{\mathrm{HBT}}}\right\} .
\end{aligned}
$$

If the unit input light is inputted to LED, the individual relative time response for LED can be get as

$$
R_{0}^{\mathrm{LED}}(t)=\int_{0}^{t} U(t-\lambda) R_{\mathrm{LED}}(\lambda) d \lambda=\left(1-e^{-\omega_{\mathrm{LED}} t}\right)
$$

At the end of the LED part, the relative response of the output signal is calculated as

$$
R(t)=R_{2}(t) \cdot R_{\mathrm{LED}}(t)=\int_{0}^{t} R_{2}(t-\lambda) R_{\mathrm{LED}}(\lambda) d \lambda
$$

where $R_{\mathrm{LED}}(\lambda)$ is $R_{\mathrm{LED}}(t)$ at time $\lambda$.

Then

$$
R(t)=\left\{\begin{array}{l}
\omega_{\mathrm{QWIP}}^{2} \omega_{\mathrm{LED}} e^{-\left(\omega_{\mathrm{HBT}}\right) t}-\omega_{\mathrm{QWIP}}^{2} \omega_{\mathrm{HBT}}+ \\
+\omega_{\mathrm{QWIP}}^{2} \omega_{\mathrm{LED}}-\omega_{\mathrm{LED}}^{2} \omega_{\mathrm{QWIP}} e^{-\left(\omega_{\mathrm{HBT}}\right) t}- \\
-\omega_{\mathrm{HBT}}^{2} \omega_{\mathrm{QWIP}}+\omega_{\mathrm{LED}}^{2} \omega_{\mathrm{QWIP}}- \\
-\omega_{\mathrm{LED}}^{2} \omega_{\mathrm{HBT}}+\omega_{\mathrm{HBT}}^{2} \omega_{\mathrm{LED}}- \\
-\omega_{\mathrm{HBT}}^{2} \omega_{\mathrm{LED}} e^{-\left(\omega_{\mathrm{QWIP}}\right) t}- \\
-\frac{\omega_{\mathrm{QWIP}} \omega_{\mathrm{HBT}}}{\omega_{\mathrm{LED}}^{2}-\omega_{\mathrm{QWIP}} \omega_{\mathrm{HBT}}} e^{-\left(\omega_{\mathrm{LED}}\right) t}
\end{array}\right\},
$$

where

$$
R_{0}=\frac{1}{\left(\omega_{\mathrm{HBT}}^{2}-\omega_{\mathrm{HBT}} \omega_{\mathrm{LED}}-\omega_{\mathrm{HBT}} \omega_{\mathrm{QWIP}}+\omega_{\mathrm{QWIP}} \omega_{\mathrm{LED}}\right)\left(\omega_{\mathrm{LED}}-\omega_{\mathrm{QWIP}}\right)}
$$

The final state of transient response of the structure can be expressed as

$$
R_{f}=\lim _{t \rightarrow \infty} R(t)=R_{0}\left\{\begin{array}{l}
-\omega_{\mathrm{QWIP}}^{2} \omega_{\mathrm{HBT}}+\omega_{\mathrm{QWIP}}^{2} \omega_{\mathrm{LED}}- \\
-\omega_{\mathrm{HBT}}^{2} \omega_{\mathrm{QWIP}}+\omega_{\mathrm{LED}}^{2} \omega_{\mathrm{QWIP}}- \\
-\omega_{\mathrm{LED}}^{2} \omega_{\mathrm{HBT}}+\omega_{\mathrm{HBT}}^{2} \omega_{\mathrm{LED}}
\end{array}\right\}=1 .
$$

The derivative of the transient response of the device is expressed by $\frac{d}{d t} R(t)$, which is a measure of the device speed, this quantity can be expressed as:

$$
\frac{d}{d t} R(t)=R_{0}\left\{\begin{array}{l}
\omega_{\mathrm{QWIP}}^{2} \omega_{\mathrm{LED}} \omega_{\mathrm{HBT}} e^{-\left(\omega_{\mathrm{HBT}}\right) t}+ \\
+\omega_{\mathrm{LED}}^{2} \omega_{\mathrm{QWIP}} \omega_{\mathrm{HBT}} e^{-\left(\omega_{\mathrm{HBT}}\right) t}+ \\
+\omega_{\mathrm{HBT}}^{2} \omega_{\mathrm{LED}} e^{-\left(\omega_{\mathrm{QWIP}}\right) t}+ \\
+\frac{\omega_{\mathrm{LED}} \omega_{\mathrm{QWIP}} \omega_{\mathrm{HBT}}}{\omega_{\mathrm{LED}}^{2}-\omega_{\mathrm{QWIP}} \omega_{\mathrm{HBT}}} e^{-\left(\omega_{\mathrm{LED}}\right) t}
\end{array}\right\} .
$$

The rise time of the considered structure is the time needed for the signal to reach the final value $R_{f}$. By using the approximation where $\omega_{\mathrm{HBT}}$ is the lowest cutoff frequency, the rise time is expressed as:

$$
\begin{aligned}
& T=\frac{-1}{\omega_{\mathrm{HBT}}} \times \\
& \times \ln \left\{\frac{H\left(1+R_{f}\right)}{\eta_{\mathrm{QWIP}} \eta_{\mathrm{HBT}} \eta_{\mathrm{LED}}\left(\omega_{\mathrm{QWIP}}^{2} \omega_{\mathrm{HBT}}-\omega_{\mathrm{HBT}}^{2} \omega_{\mathrm{QWIP}}\right)}\right\},
\end{aligned}
$$

where

$$
\begin{aligned}
& H=-\omega_{\mathrm{QWIP}}^{2} \omega_{\mathrm{HBT}}+\omega_{\mathrm{QWIP}}^{2} \omega_{\mathrm{LED}}-\omega_{\mathrm{HBT}}^{2} \omega_{\mathrm{QWIP}}+ \\
& +\omega_{\mathrm{LED}}^{2} \omega_{\mathrm{QWIP}}-\omega_{\mathrm{LED}}^{2} \omega_{\mathrm{HBT}}+\omega_{\mathrm{HBT}}^{2} \omega_{\mathrm{LED}} .
\end{aligned}
$$

\section{Results and discussions}

The device parameters used in the following calculations are as follow, $\omega_{\mathrm{QWIP}}=1 \mathrm{GHz}, \quad \omega_{\mathrm{HBT}}=0.1 \mathrm{GHz}$, $\omega_{\mathrm{LED}}=1.2 \mathrm{GHz}$ and $\eta_{\mathrm{QWIP}} \eta_{\mathrm{HBT}} \eta_{\mathrm{LED}}=1$. The input infrared radiation is assumed as a step function in time. The first set of curves shown in Fig. 2a represents the transient behavior of the developed structure where $\omega_{\mathrm{HBT}}$ is chosen to be the smallest cutoff frequency. The solid line curve represents the exact response and the dotted line curve corresponds to the first order dominant pole approximation concerning HPT, while the dashed line curve corresponds to the first order dominant pole approximation for LED, and the dashed dotted line indicates the QWIP approximation. It is clear from the 
figure the exact response of the considered structure is in close with the first order HPT response, this is because $\omega_{b}=0.1 \mathrm{GHz}$ is close to zero in the pole-zero map than $\omega_{\text {QWIP }}$ and $\omega_{\text {LED }}$ as in Fig. $2 b$.

Fig. 3 shows variation of the output derivative with time (which measures the speed of the output changes) at the three approaches of the constituent devices. It is clear that the cutoff frequency of the device plays a major role in determining the response speed, since the large value of cutoff frequency yields the device to arrive to its final state quickly unlike those with a smaller value. The output derivative concerning both QWIP and LED decreases with time, while nearly increases with time as regarding HPT and all the structure. The decrease with time for both QWIP and LED is caused by their higher cutoff frequencies, which helps to reduce the arrival time to their final state. The output derivative concerning HPT is similar to the output derivative of all structure due to the closest HPT pole to zero than the other concerning both QWIP and LED as in Fig. 2b.

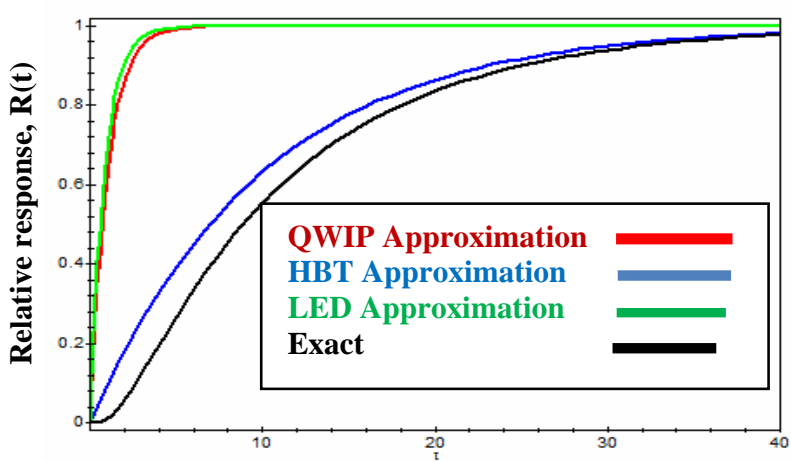

Time t, ns

Fig. 2a. Relative transient response for constituent devices approximation and exact response at $\omega_{b}=0.1 \mathrm{GHz}$.

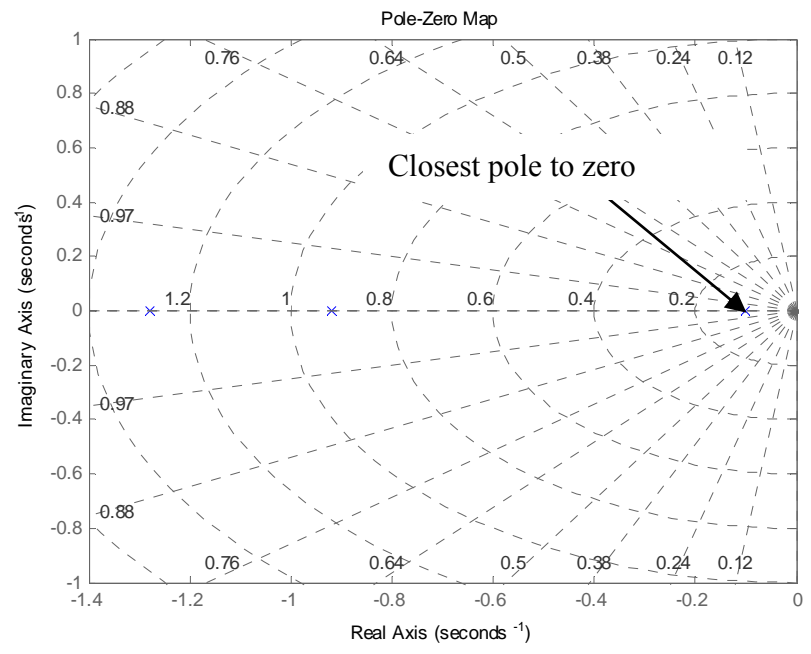

Fig. 2b. P-Z Map for $\omega_{\mathrm{HBT}}=0.1 \mathrm{GHz}, \omega_{\mathrm{QWIP}}=1 \mathrm{GHz}$, $\omega_{\text {LED }}=1.2 \mathrm{GHz}$

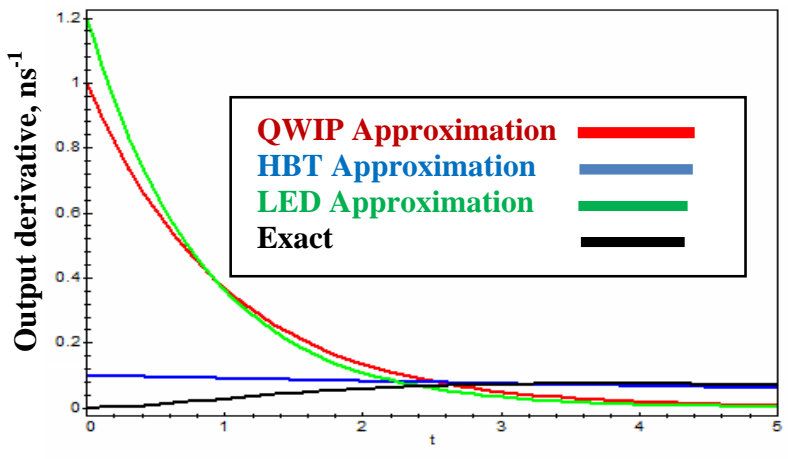

Time t, ns

Fig. 3. Output derivative for constituent devices approximation and exact response at $\omega_{b}=0.1 \mathrm{GHz}$.

When there is no negative pole close to zero as in Fig. $4 \mathrm{a}$, there is no similarity in response among the constituent devices with the response of all the structure. This fact is clear in Fig. 4b, where the cutoff frequencies concerning QWIP, HBT, and LED are close to each other so they have the same and close behavior.

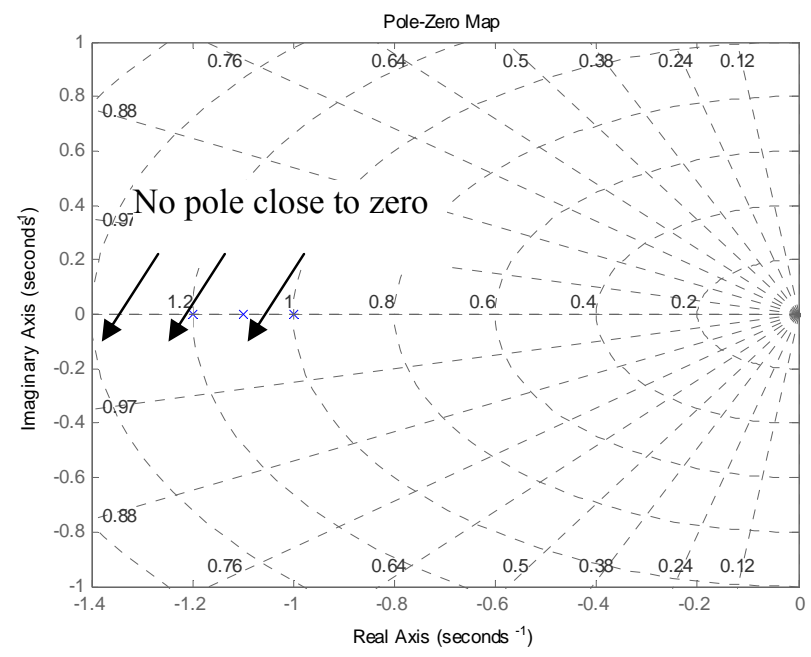

Fig. 4a. P-Z Map for $\omega_{\mathrm{HBT}}=1.1 \mathrm{GHz}, \omega_{\mathrm{QWIP}}=1 \mathrm{GHz}$, $\omega_{\mathrm{LED}}=1.2 \mathrm{GHz}$.

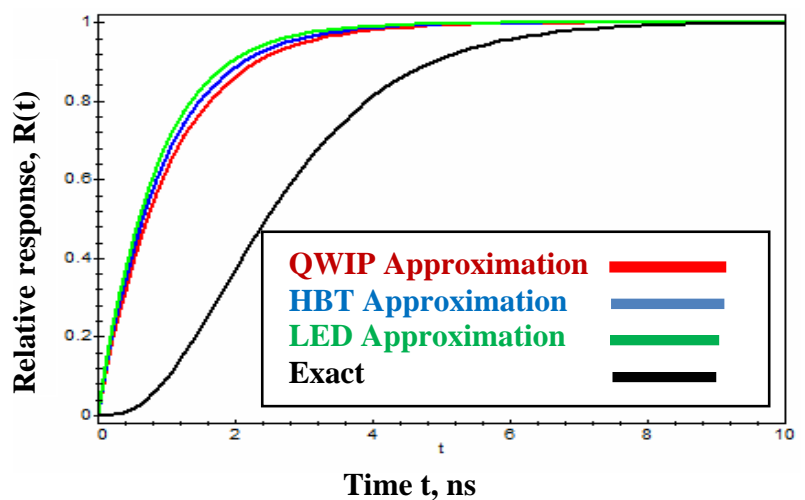

Fig. 4b. Relative transient response for constituent devices approximation and exact response at $\omega_{b}=1.1 \mathrm{GHz}$. 
Also, the output derivative itself exihibits a similar and close behavior as concerning QWIP, HBT, and LED due to their nearby cutoff frequencies as clear in Fig. 5. It is shown from the figure the output derivative increases with time because it takes larger time to reach its final value due to the overall delay time through the layers of QWIP, HBT, and LED.

The final state value depends on the quantum efficiencies of QWIP, HBT, and LED, where it is assumed to be $\eta_{\mathrm{QWIP}} \eta_{\mathrm{HBT}} \eta_{\mathrm{LED}}=1$ in the proposed model. When this final value increases the device needs more time to catch it, hence the rise icreases as illustrated in Fig. 6. It is shown that the cutoff frequency plays a major role in controlling the rise time, also the rise time behavior for HBT is again in close proximity to the rise time behavior of all the structure. If the value of cutoff frequency of HBT is increased to close the cutoff frequency for both QWIP and LED, the rise time behavior of the overall structure is quitely different from the other constituent devices as illustrated in Fig. 7.

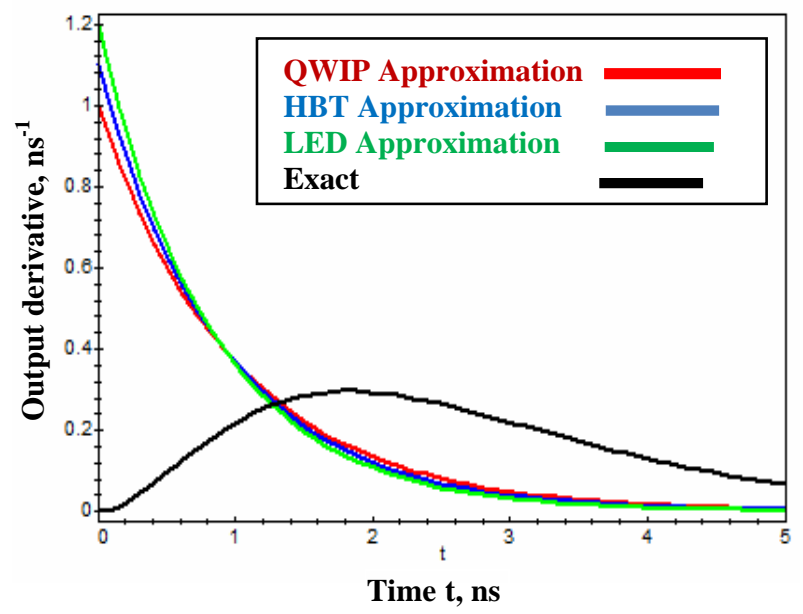

Fig. 5. Output derivative for constituent devices approximation and exact response at $\omega_{\mathrm{b}}=1.1 \mathrm{GHz}$.

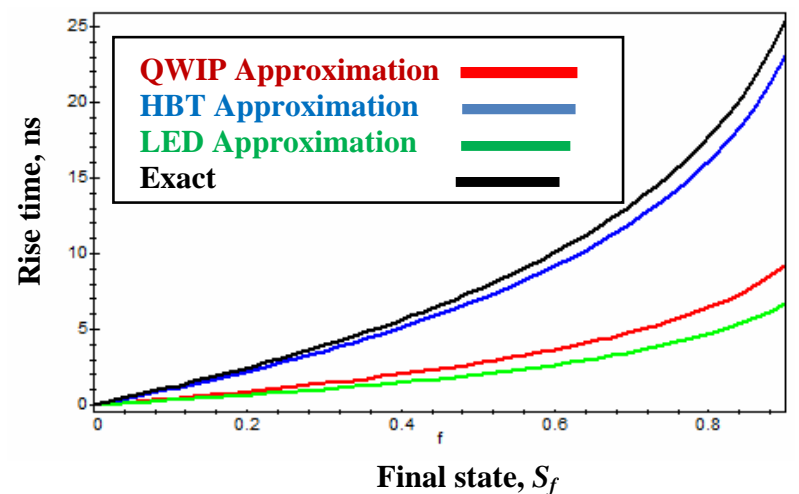

Fig. 6. Rise time versus final state $S_{f} \omega_{b}=0.1 \mathrm{GHz}$.

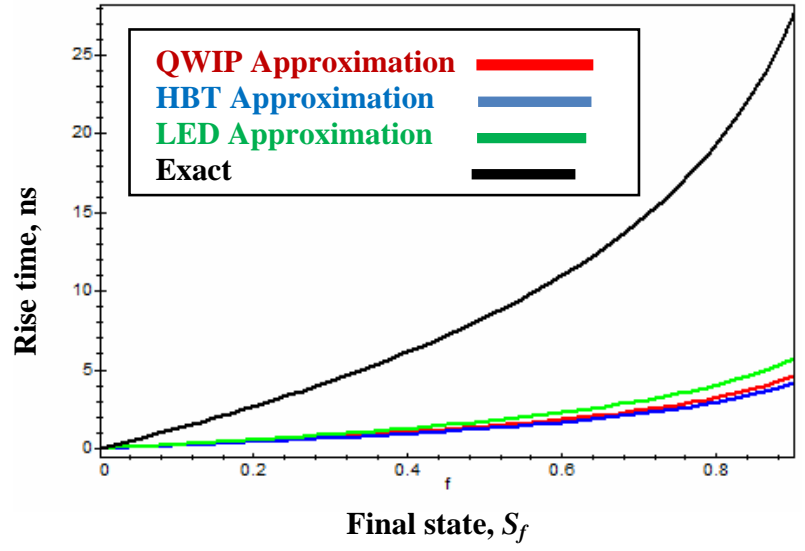

Fig. 7. Rise time versus final state $S_{f}$ at $\omega_{b}=1.1 \mathrm{GHz}$.

\section{Conclusion and future work}

Analytical modeling concerning the temporal behavior of a quantum well structure optical integrated device has been developed in this paper. The structure contains a quantum well infrared photodetector to detect the signal, a heterojunction phototransistor for amplifying, and a light emitting diode for emitting visible light range. This modeling is based on applying dominant pole approximation scheme and convolution theorem. The analytical expressions concerning all identities of transient response are determined. The results show that the transient behavior of the developed structure is in close proximity with the constituent device which has the smallest cutoff frequency. The quantum efficiency of any constituent device causes an enhancement in the transient response. Also, the constituent device with a higher cutoff frequency has no effect on both the rise time and the output derivative of the device. As a future extension to this study, the use of a resonant cavity to modeling this device is planned to show the effect of all interesting parameters on its dynamic behavior.

\section{References}

1. Lu W, Liu Z L, Zhen H L, et al. A detector for infrared near-infrared wavelength up-conversion. PRC Patent, 200610028489.9,

2. Qasaimeh O., Zhou W., Bhattacharya P. et al. Monolithically integrated low-power phototransceivers for optoelectronic parallel sensing and processing applications. J. Lightwave Technology. 2001. 19, No. 4. P. 546-552.

3. Ryzhii V., Liu H.C., Khmyrova I., and Ryzhii M. Analysis of integrated quantum-well infrared photodetector and light-emitting diode for implementing pixelless imaging device. IEEE $J$. Quant. Electron. 2007. 33. P. 1527-1531. 
4. Zhen H.L., Xiong D.Y., Zhou X.C. et al. Study on the $p$-type QWIP-LED device. Sci. China Ser. G: Phys. Mech. Astron. 2006. 36. P. 401-410.

5. PRC Patent, 200610028489.9. A detector for infrared-near-infrared wavelength up-conversion. W. Lu, Z.L. Liu, H.L. Zhen et al. 2006-06-30.

6. L. Wu and W. Shen, Resonant-cavity-enhanced farinfrared upconversion imaging devices. IEEE $J$. Quantum Electron. 2007. 43, No. 5. P. 411-418.

7. Ding Y., Meriggi L., Steer M. et al. Design, simulations, and optimizations of mid-infrared multiple quantum well LEDs. J. Procedia Eng. 2016. 140. P. 36-42.

8. Sun J., Choi K., and Olver K. Fabrication of resonator-quantum well infrared photodetector test devices. J. Micro/Nanolith. MEMS MOEMS. 2014. 13. P. 013004.

9. Sun J. and Choi K. Fabrication of resonatorquantum well infrared photodetector focal plane array by inductively coupled plasma etching. $J$. Opt. Eng. 2016. 55, No. 2. P. 026119.

10. Eladl Sh., Nasr A., and Aboshosha A. Dynamic characteristics of QWIP-HBT-LED optoelectronic integrated devices. Semiconductor Physics, Quantum Electronics \& Optoelectronics. 2009. 12, No. 3. P. 260-263.

11. Mishra R., Saxena R., and Jain R. Application of FRFT convolution theorem in filtering. Intern. $J$. Information Eng. 2013. 3, Issue 3, P. 71-75. 